\title{
Solvation Free Energy of Self-Assembled Complexes: Using Molecular Dynamics to Understand the Separation of ssDNA-Wrapped Single-Walled Carbon Nanotubes ${ }^{\dagger}$
}

Kevin R. Hinkle ${ }^{1,2^{*}}$, Frederick R. Phelan, Jr. ${ }^{1 *}$

${ }^{1}$ Materials Science and Engineering Division, National Institute of Standards and Technology, Gaithersburg, Maryland

${ }^{2}$ Department of Chemical and Materials Engineering, University of Dayton, Dayton, $\mathrm{OH}$

*Corresponding Authors: hinklek1@udayton.edu; frederick.phelan@nist.gov

\section{Supplementary Information}

Application of FEP Technique to ssDNA-SWCNT Complex

An additional difficulty that arises during FEP application lies in the fact that these complexes are not stabilized by any sort of covalent bonds between the ssDNA and SWCNT, only by the $\pi-\pi$ stacking interactions of the aromatic nucleobases with the aromatic carbon surface and the hydrophobic forces that cause the ssDNA to assume configurations where its charged phosphate backbone interfaces with the solvating water. During the FEP process, as the ssDNA is decoupled from the both the solvent molecules and the SWCNT, these hydrophobic forces and $\pi-\pi$ stacking interactions disappear, allowing the ssDNA oligomers to diffuse off the carbon surface thereby leading to the breakdown of the complex. This dissociation can be observed by measuring the SASA of the SWCNT (see Figure S1). 


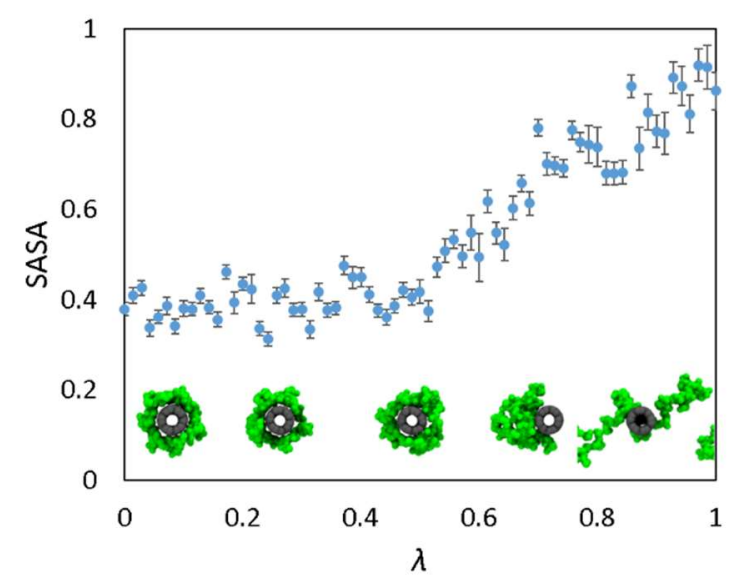

Figure S1. Breakdown ssDNA-SWCNT complex during decoupling with unrestrained DNA. Each blue point is the average relative SASA of the SWCNT at a particular value of $\lambda$. The upturn in relative $S A S A$ reveals an increasing degree of dissociation. Inset) Representative snapshots from simulations at $\lambda$ values of $0,0.25,0.5,0.75$, and 1.0 .

Another detail, which has thus far been implied, deals with the packing conformations of the ssDNA. It is hypothesized that the same complex can present different solvation energies if the arrangement of the ssDNA is significantly different. This means that representative wrapping configurations must be selected on some basis and then maintained throughout the entire free energy pathway. In order to solve both of these problems, it will be necessary to apply position restraints on the ssDNA as it is decoupled from the solvent and the SWCNT. Such position restraints have again been used in the field of computer-aided drug design (CADD) to localize ligands within their bonding pocket during decoupling ${ }^{1}$. Additionally, they have been applied to hydrating water molecules to determine the role they contribute to proper ligand binding ${ }^{2}$. In the current work, these restraints are applied to each heavy atom in the ssDNA strand and take the form of a simple harmonic potential (Eq. (1)); however, one must take care in the selection of the force constant, $k$. A value that is too high results in a stronger restraint and subsequently an undersampling of the relevant conformational space. Conversely, a value that is too low results in weaker restraints that may allow for needless or even undesirable fluctuations in the wrapping structure during the decoupling of the ssDNA.

$$
U_{i}=\frac{k}{2}\left(r_{i}-r_{i 0}\right)^{2}
$$


Hamelburg and McCammon ${ }^{38}$ have presented a method by which the restraining force constant can be systematically determined by allowing the system to evolve in an unrestrained manner and measuring the average fluctuation in atomic positions, $\left\langle\delta r^{2}\right\rangle$. Once this quantity is known, the maximum value for $k$ that will still permit average fluctuations of a similar size can be found using Eq. (2).

$$
k=\frac{3 R T}{\left\langle\delta r^{2}\right\rangle}
$$

This approach will allow for the calculation of the relative solvation energy of the complexed structures, but only for the specific adsorbed conformation to which it is restrained. Therefore, it is predicted that care must be taken when selecting these initial configurations.

\section{Selection of Representative Wrapping Structures}

After the generation of an ensemble of equilibrated conformations via REMD simulation, it is necessary to select certain conformations for further analysis in the FEP scheme. This is done via the application of the gromos clustering algorithm with a root-mean-square displacement cutoff of $1.0 \mathrm{~nm}$ following which the center conformations of the largest three (3) clusters were chosen as representative wrappings (Figures $\mathbf{S 2 a}, \mathbf{S 2 b}, \mathbf{S 2 c}$ ). In order to verify that the application of position restraints does not lead to significant under-sampling of the conformational space, the selected frames from the REMD ensemble were used as the initial condition for further $10 \mathrm{~ns}$ unrestrained standard MD simulations (single replica) and the root mean square displacement (RMSD) of the ssDNA was monitored (Figure S2d). A subsequent clustering analysis on this unrestrained trajectory resulted in a single cluster. These two results indicate that the adsorbed wrappings do not change appreciably over the simulation time at $300 \mathrm{~K}$ (Figure S2e) and that restraining the ssDNA to maintain a certain wrapping conformation is a valid approach.

These unrestrained trajectories also allowed for the estimation of a proper restraining constant to be used during the FEP simulations as discussed above. Calculation of the atomic root mean square fluctuation (RMSF) for all phosphorous atoms in the ssDNA backbone was found to equal $0.0826 \pm 0.0179 \mathrm{~nm}$. Application of Eq. (2) results in a restraining coefficient, $k=1096 \pm$ $237 \mathrm{~kJ} / \mathrm{mol} \cdot \mathrm{nm}^{2}$. Subsequently, during all FEP simulations in which position restraints were 
enacted, a value of $800 \mathrm{~kJ} / \mathrm{mol} \cdot \mathrm{nm}^{2}$ was used as the coefficient to ensure proper sampling of the atomic fluctuations.
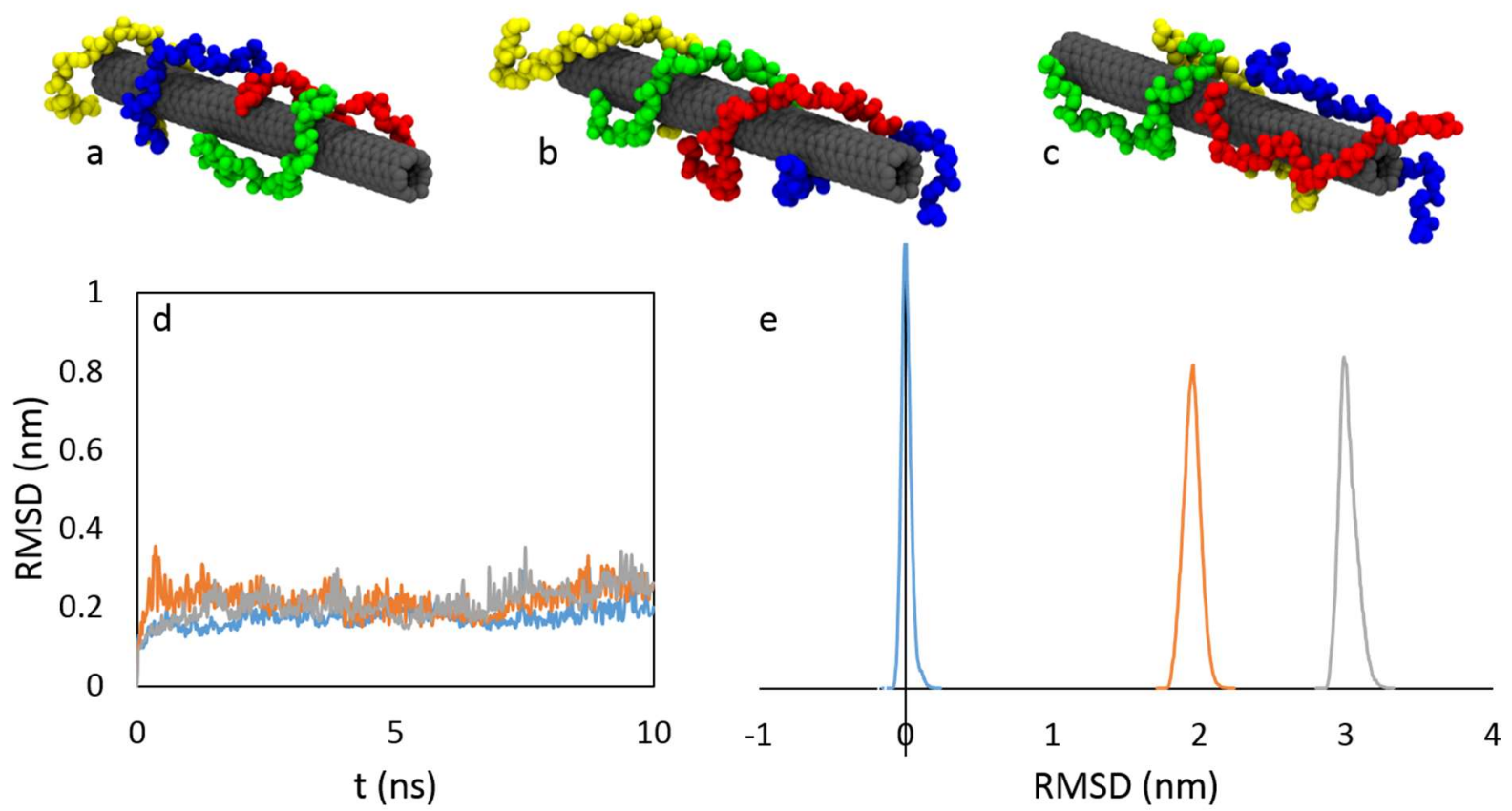

Figure S2. Unrestrained MD simulations do not result in large conformational changes of the adsorbed ssDNA indicating that applying position restraints will not lead to significant undersampling; $a, b$, and c) Three representative conformations of identical sequence and chirality chosen via clustering analysis; d) RMSD of the ssDNA during unrestrained simulations; e) distributions of the RMSD with respect to the conformation in (a) demonstrating the magnitude of the differences between the three initial conditions and that they remain separate in unrestrained simulations.

Table S2. Relative free energies for $(T A T)_{4} /(6,5)-S W C N T$ with increasing ssDNA loading. Uncertainties represent the standard deviation of $N=3$ different configurations.

\begin{tabular}{cc} 
Number ssDNA, $\boldsymbol{i}$ & $\boldsymbol{\Delta} \mathbf{G}_{\mathbf{0} \rightarrow \mathbf{i}} \frac{\boldsymbol{k J}}{\boldsymbol{m o l}}$ \\
\hline 1 & $-6958 \pm 106$ \\
2 & $-13971 \pm 147$ \\
3 & $-21282 \pm 227$ \\
4 & $-28841 \pm 115$
\end{tabular}



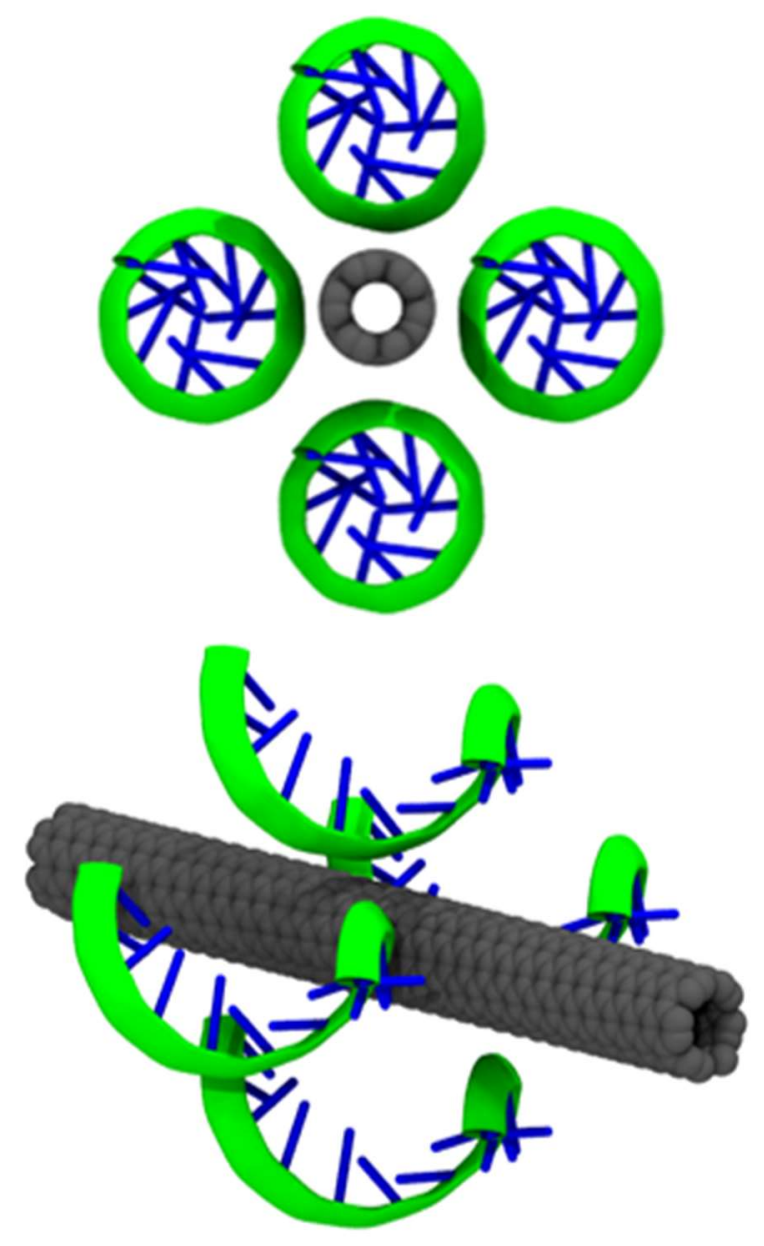

Figure S3. Representative initial ssDNA/SWCNT configuration. Note the ideal helix structure of the ssDNA backbone. Top) End view. Bottom) Rotated view.

\section{References}

(1) Wang, J.; Deng, Y.; Roux, B. Absolute Binding Free Energy Calculations Using Molecular Dynamics Simulations with Restraining Potentials. Biophys. J. 2006, 91 (8), 2798-2814. https://doi.org/10.1529/biophysj.106.084301.

(2) Hamelberg, D.; McCammon, J. A. Standard Free Energy of Releasing a Localized Water Molecule from the Binding Pockets of Proteins: Double-Decoupling Method. J. Am. Chem. Soc. 2004, 126 (24), 7683-7689. https://doi.org/10.1021/ja0377908. 